\title{
Increased markers of thrombogenesis in chronic atrial fibrillation: effects of warfarin treatment
}

\author{
Gregory Y H Lip, Gordon D O Lowe, Ann Rumley, Francis G Dunn
}

\begin{abstract}
Objective-To determine whether chronic atrial fibrillation is associated with abnormalities in plasma fibrinogen, von Willebrand factor (vWF) (a marker of endothelial disturbance), or fibrin Ddimer (a measure of fibrin turnover); and if so, whether such levels are related to haemodynamic disturbance (enlarged left atrium, poor left ventricular function) or existing treatment with warfarin or aspirin. To investigate the effects of introducing warfarin in patients with atrial fibrillation on fibrinogen and Ddimer levels.
\end{abstract}

Design-Cross sectional population sample controlled study and longitudinal study of patients undergoing anticoagulation.

Setting-District general hospital.

Subjects-87 patients (44 men and 43 women of mean (SEM) age $63.0(1 \cdot 0)$ ) with chronic atrial fibrillation. At the time of the study, 37 were taking no antithrombotic medication (group 1), 31 were taking warfarin (including two on warfarin and aspirin) (group 2) and 19 were taking aspirin alone (group 3 ). They were compared with 158 population controls from a random population sample (the second Glasgow monitoring trends and determinants in cardiovascular disease study). As part of clinical treatment warfarin was introduced in 20 patients with chronic atrial fibrillation (14 men and six women of mean (SEM) (range) age 63.9 (2.35 (32-74) years).

Results-Plasma fibrinogen remained significantly increased in patients of group 1 (no antithrombotic medication) compared with that of the population controls (median difference $1.23 \mathrm{~g} / 1 ; 95 \%$ confidence interval (CI) 0.88 to $1.62, \mathrm{P}<$ $0 \cdot 0001)$. There was also a significant increase in plasma D-dimer levels (median difference $77 \mathrm{ng} / \mathrm{ml} ; 95 \%$ CI 38 to $122, P<0.01$ ) and vWF (median difference $63 \mathrm{IU} / \mathrm{dl}$; $95 \%$ CI 38 to $89, \mathrm{P}<$ $0.0001)$. There was no significant difference in plasma fibrinogen (median difference $0.14 \mathrm{~g} / 1 ; 95 \% \mathrm{CI}-0.44$ to $0.77, \mathrm{P}$ $=0.65$ ) or $\mathrm{vWF}$ (median difference 3.5 IU/d1; $95 \%$ CI -41 to $41, P=$ not significant in patients of group 2 (warfarin treatment) compared with that of patients in group 1. Levels of D-dimer were significantly lower in group 2 (median difference $90 \mathrm{ng} / \mathrm{ml}, 95 \%$ CI 39 to $150, P<0.0001$ ) than in group 1 . There were no significant differences in plasma fibrinogen (median difference $0.08 \mathrm{~g} / \mathrm{l}$; $95 \% \mathrm{CI}-0.52$ to $0.77, P=0.73$ ), $\mathrm{D}$-dimer (median difference $-34 \mathrm{ng} / \mathrm{ml}$; 95\% CI -114 to $21 \cdot 0, P=0.25$ ), or vWF (median difference $2 \%$; $95 \% \mathrm{CI}-35$ to $41, P=$ not significant) levels between patients of groups 1 and 3. There were no significant correlations between the coagulation indices and left atrial volume or ventricular function. There was a significant positive correlation between plasma fibrin D-dimer and vWF levels in patients of groups 1 and $3(r=0.52, P<0.001)$. There was a significant reduction in median plasma fibrin D-dimer levels at 2 months after the introduction of warfarin (181 ng/ml v $80 \mathrm{ng} / \mathrm{ml}, P<0.001$ ), but no effect on plasma fibrinogen.

Conclusions-Increased median plasma fibrinogen and vWF levels were found in patients with chronic atrial fibrillation. Plasma D-dimer levels were also increased in patients with chronic atrial fibrillation not receiving warfarin, suggesting increased intravascular thrombogenesis in such patients. Introduction of warfarin normalised circulating fibrin Ddimer levels, suggesting that warfarin treatment was effective in preventing excessive fibrin turnover, consistent with the antithrombotic effects of warfarin. These results suggest three possible thrombotic markers to assess patients with atrial fibrillation who are at high risk of thrombogenesis; D-dimer also merits assessment as a measure of reduction in thrombotic risk in patients receiving warfarin.

(Br Heart f 1995;73:527-533)

Keywords: atrial fibrillation; warfarin; thrombotic markers

The mechanisms of increased thromboembolic risk in patients with atrial fibrillation have not been fully elucidated. Abnormalities in plasma coagulation factors indicative of a prothrombotic state may in part account for this risk. Increased levels of plasma fibrinogen are associated with an increased risk of ischaemic heart disease and stroke ${ }^{1-3}$ and may promote disease by increasing fibrin 
formation and platelet aggregation, or by rheological effects (blood viscosity, red cell aggregation) which may promote disturbed blood flow. Plasma von Willebrand factor (vWF) is a marker of endothelial disturbance which is associated with thromboembolic stroke, ${ }^{4}$ ischaemic heart disease, and peripheral arterial disease. ${ }^{56}$ Plasma fibrin D-dimer levels are increased in venous thrombosis, ${ }^{7}$ peripheral arterial disease, ${ }^{5}$ and in patients at risk of ischaemic heart disease. ${ }^{6}$ In addition, two small studies have suggested that plasma levels of D-dimer are increased in patients with atrial fibrillation, irrespective of whether or not co-existing structural heart disease is present, compared with those in patients in sinus rhythm. ${ }^{89}$

Recent major, prospective studies have demonstrated that warfarin reduces the incidence of thromboembolic complications in patients with atrial fibrillation; the effect of aspirin, however, is less certain. ${ }^{10}$ The effect of the introduction of warfarin treatment in plasma prothrombotic indices in patients with atrial fibrillation has not been previously reported. The present study was therefore performed to determine whether chronic atrial fibrillation is associated with abnormalities in plasma fibrinogen, vWF, or D-dimer levels; and if so, whether such levels are related to measurable haemodynamic disturbance (enlarged left atrium, poor left ventricular function) or existing treatment with warfarin or aspirin. We subsequently investigated the effects of introducing warfarin (as part of clinical management) in patients with atrial fibrillation on fibrinogen and D-dimer levels.

\section{Patients and methods}

CROSS-SECTIONAL POPULATION CONTROLLED STUDY

Patients with chronic atrial fibrillation were recruited from outpatient clinics within, and inpatients attending, Stobhill General Hospital. Inpatients were usually referrals from patients electively admitted for nonacute surgical conditions (for example, hernia, varicose veins) who were identified before operation. Chronic atrial fibrillation was defined as atrial fibrillation documented electrocardiographically on at least two occasions, separated by a period of 6 or more weeks at review performed by the patient's general practitioner, or in the hospital or outpatient clinic. Patients more than 75 years and those with significant systemic illness, (which influences fibrinogen, vWF, and fibrin D-dimer levels) such as renal failure, liver impairment (defined as abnormal liver function tests with aspartate transaminase or alanine transaminase levels more than twice the upper limit of normal), acute or chronic infection, or neoplastic disease, were excluded. Patients with hypertension, acute cardiovascular or cerebrovascular syndromes (angina, myocardial infarction, stroke), limited venous access, and those requiring transfusion or actively bleeding were also excluded.

Blood samples were drawn from the ante- cubital vein by careful venepuncture, anticoagulated with trisodium citrate $(0 \cdot 11 \mathrm{M}, 9: 1$ $\mathrm{vol} / \mathrm{vol}$ ), and centrifuged. The platelet-free plasma was immediately separated and frozen at $-40^{\circ} \mathrm{C}$. Plasma D-dimer antigen levels were measured using an enzyme linked immunosorbent assay (ELISA) (AGEN, Parsippany, New Jersey). Plasma fibrinogen was assayed by the Clauss assay using a CoagA-mate coagulometer and Organon Teknika reagents and standards (Organon Teknika, Cambridge). Plasma vWF was assayed using an ELISA (DAKO, Copenhagen). The sensitivity of the assays allowed measurement of plasma D-dimer, $\mathrm{vWF}$, and fibrinogen levels to a minimum of $30 \mathrm{ng} / \mathrm{ml}, 1 \mathrm{IU} / \mathrm{dl}$, and 0.5 $\mathrm{g} / \mathrm{l}$ respectively. The reproducibility of all three methods allowed inter-assay coefficients of variation of less than $5 \%$. Results were compared with those of 158 population controls aged 55-64 years in sinus rhythm sampled from a local population survey (second north Glasgow monitoring trends and determinants in cardiovascular disease study ${ }^{11}$ (unpublished observations (for D-dimer and vWF levels)).

Two dimensional and Doppler echocardiography were performed using a Hewlett-Packard Sonos 500 machine (Hewlett-Packard, Arondale, Pennsylvania) equipped with a standard $2.5 \mathrm{MHz}$ transducer and off line analytical methodology was used to quantify measurements. Left and right atrial volumes were calculated using a standard ellipsoid formula. ${ }^{12}$ Left ventricular dimensions were measured using $M$ mode echocardiography in the parasternal long axis view ${ }^{13}$ and the fractional shortening calculated as an index of left ventricular function.

\section{EFFECTS OF INTRODUCING WARFARIN}

All patients given warfarin as part of the clinical management were managed on an outpatient basis. Clinical examination and echocardiography were performed and baseline blood samples taken at their initial visit. Blood samples, including determination of an international normalised ratio (INR) of the prothrombin time to establish the intensity of the effect of warfarin, aiming for a target INR of 2.0-3.0 were subsequently taken on days 3 , 5 , and 7 and 2 months after the introduction of warfarin. The study was approved by the hospital ethics committee.

All haematological values and cardiac dimensions were expressed as median (interquartile range) (IQR). Statistical comparison was performed using the $\chi^{2}$ test, analysis of variance and $t$ tests for normal distributions and the Kruskal-Wallis test and Mann-Whitney two sample rank test (for nonparametric distributions), and $95 \%$ confidence intervals (CI) were calculated for the point estimate of the difference between medians. Statistical comparison of sequential results was performed using the two sample paired Wilcoxon test for non-parametric distributions. Correlations between clinical variables or rheological measurements were performed using Spearman's rank correlation 
Table 1 Patient characteristics (cross-sectional study)

\begin{tabular}{|c|c|c|c|c|}
\hline & \multicolumn{3}{|c|}{ Patients with chronic atrial fibrillation } & \multirow[b]{2}{*}{$\begin{array}{l}\text { Controls } \\
\text { (sinus rhythm) }\end{array}$} \\
\hline & $\begin{array}{l}\text { Group } 1 \\
\text { (no antithrombotic } \\
\text { treatment) }\end{array}$ & $\begin{array}{l}\text { Group } 2 \\
\text { (administered } \\
\text { warfarin) }\end{array}$ & $\begin{array}{l}\text { Group } 3 \\
\text { (administered } \\
\text { aspirin) }\end{array}$ & \\
\hline $\begin{array}{l}\text { No. of patients } \\
\text { No. of men }(\%) \\
\text { Smokers }(\%) \\
\text { Mean (SEM) age (years) }\end{array}$ & $\begin{array}{l}37 \\
19(51) \\
12(32) \\
62 \cdot 6(1 \cdot 6)\end{array}$ & $\begin{array}{l}31 \\
12(39) \\
7(23) \\
60 \cdot 3(1 \cdot 6)\end{array}$ & $\begin{array}{l}19 \\
13(68) \\
3(16) \\
68 \cdot 4(1 \cdot 6)\end{array}$ & $\begin{array}{l}158 \\
88(56) \\
64(41) \\
59 \cdot 3(0 \cdot 2)\end{array}$ \\
\hline
\end{tabular}

method. Stepwise multiple regression analyses were performed with plasma fibrinogen, vWF, and fibrin $\mathrm{D}$-dimer as the dependent variables to determine whether age, smoking habit, left atrial volume (ellipsoid), right atrial volume, left ventricular fractional shortening, or body mass index contributed significantly to levels of these coagulation indices. $P \leqslant 0.05$ was considered significant.

\section{Results}

Eighty seven patients with chronic atrial fibrillation (44 men and 43 women of mean (SEM) age $63.0(1.0)$ years) were enrolled in the cross-sectional study. At the time of study, 37 were receiving no antithrombotic treatment (group 1), 31 were taking warfarin (including two on warfarin and aspirin) (group 2), and 19 were taking aspirin alone (group 3). Table 1 gives details of the age, sex, and smoking habit of these patients and the 158 population controls. There were no significant differences in sex ratio $\left(\chi^{2}=4 \cdot 79\right.$, $3 \mathrm{df}, \mathrm{P}=0 \cdot 19)$ or proportions of smokers $\left(\chi^{2}=\right.$ $7.39,3 \mathrm{df}, P=0.06$ ) between the groups studied, although patients with atrial fibrillation tended to smoke less than controls. Patients in group 3 were also older than those in other groups (one way analysis of variance, $P<0.01)$. Sixty seven of the 87 patients had a predisposing factor to atrial fibrillation. Valvular heart disease was the most common factor $(n=34)$ followed by ischaemic heart disease $(n=30$, including 10 patients with a clinical history of valvular heart disease and ischaemic heart disease). Other causes included cardiomyopathy $(n=3)$ but 20 patients did not have any obvious predisposing factors.

In the longitudinal study warfarin treatment was introduced in 20 patients with chronic atrial fibrillation (14 men and six women of mean (SEM) range) age 63.9 $(2 \cdot 35)(32-74)$ years $)$.

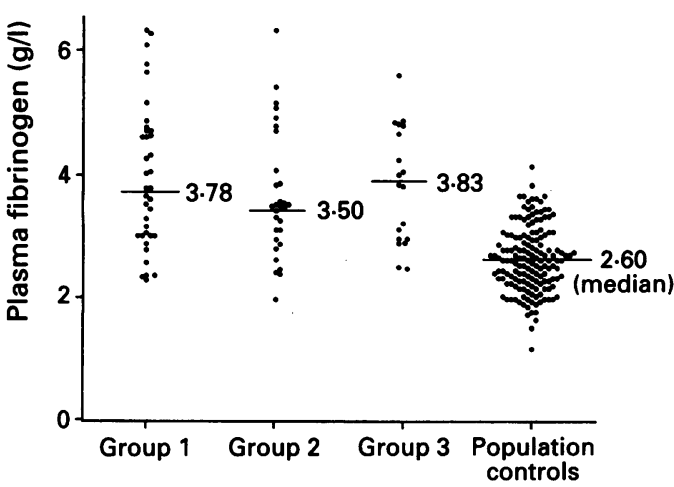

Figure 1 Plasma fibrinogen and chronic atrial fibrillation. Group 1, patients with atrial fibrillation not receiving antithrombotic treatment; group 2, patients with atrial fibrillation receiving warfarin; group 3, patients with atrial fibrillation receiving aspirin.

\section{CROSS-SECTIONAL STUDY}

Fibrinogen levels were measured in all 87 patients (median (IQR) 3.58 (3.0-4.7) g/l) but because of technical problems levels of plasma D-dimer were measured in only 81 patients (median (IQR) 89 (50-193) $\mathrm{ng} / \mathrm{ml}$ and levels of vWF in only 67 patients median (IQR) 158 (114-200) IU/dl). As a whole the correlations between plasma fibrinogen and D-dimer $(r=0.23, P=0.03)$ and between vWF and D-dimer $(r=0.29, P=0.01)$ were weak.

Plasma fibrinogen remained significantly increased in patients of group 1 (no antithrombotic treatment) compared with that of the population controls (median difference $1.23 \mathrm{~g} / 1 ; 95 \%$ confidence interval (CI) 0.88 to $1.62, \mathrm{P}<0.0001$ ) (table 2 , fig 1 ). There were also significant increases of plasma D-dimer levels (median difference 77 $\mathrm{ng} / \mathrm{ml} ; 95 \% \quad$ CI 38 to $122 \quad \mathrm{P}<0.0001)$ and vWF (median difference $63 \mathrm{IU} / \mathrm{dl} ; 95 \%$ CI 38 to $89, P<0.0001$ ) in these patients compared with those in the controls (table 2 , figs 2 and 3 ).

There was no significant difference in plasma fibrinogen (median difference $0.14 \mathrm{~g} / \mathrm{l}$; $95 \% \mathrm{CI}-0.44$ to $0.77, \mathrm{P}=0.65$ ) or $\mathrm{vWF}$ values of patients in group 2 (warfarin treatment) (median difference $3.5 \mathrm{IU} / \mathrm{dl} ; 95 \% \mathrm{CI}$ -41 to $41, P=$ not significant) compared with those of patients in group 1 (table 2). Plasma D-dimer levels, however, were significantly lower in group 2 than in group 1 (median difference $90 \mathrm{ng} / \mathrm{ml}, 95 \%$ CI 39 to $150, \mathrm{P}<0.0001$ ) (table 2).

There were no significant differences in

Table 2 Plasma fibrinogen, von Willebrand factor and fibrin D-dimer in chronic atrial fibrillation

\begin{tabular}{|c|c|c|c|c|}
\hline & \multicolumn{3}{|c|}{ Patients with chronic atrial fibrillation } & \multirow[b]{2}{*}{$\begin{array}{l}\text { Controls } \\
\text { (sinus rhythm) }\end{array}$} \\
\hline & $\begin{array}{l}\text { Group } 1 \\
\text { (no antithrombotic } \\
\text { treatment) }\end{array}$ & $\begin{array}{l}\text { Group } 2 \\
\text { (administered } \\
\text { warfarin) }\end{array}$ & $\begin{array}{l}\text { Group } 3 \\
\text { (administered } \\
\text { aspirin) }\end{array}$ & \\
\hline \multirow{2}{*}{$\begin{array}{l}\text { Fibrinogen }(\mathrm{g} / \mathrm{l}) \\
\text { D-dimer (ng/ml) } \\
\text { von Willebrand } \\
\text { factor (IU/dl) }\end{array}$} & $\begin{array}{c}3 \cdot 78(3 \cdot 00-4 \cdot 7)^{\star} \\
158(81-292)^{\star}\end{array}$ & $\begin{array}{c}3 \cdot 50(2 \cdot 95-4 \cdot 70) \\
50(50-60)^{\star \star}\end{array}$ & $\begin{array}{c}3 \cdot 83(2 \cdot 92-4 \cdot 77) \\
102(77-155)\end{array}$ & $\begin{array}{l}2 \cdot 60(2 \cdot 24-2 \cdot 99) \\
76(53-103)\end{array}$ \\
\hline & $152(108-198)^{\star}$ & $169(118-227)$ & $161(118-196)$ & $105(80-147)$ \\
\hline
\end{tabular}


Table 3 Effects of introducing warfarin

\begin{tabular}{llllll}
\hline & Pre-warfarin & Day 3 & Day 5 & Day 7 & 2 months \\
\hline D-dimer $(\mathrm{ng} / \mathrm{ml}$ & $181(111-235)$ & $136(90-200$ & $173(125-244)$ & $130(92-209)$ & $80(61-137)$ \\
Fibrinogen $(\mathrm{g} / \mathrm{l})$ & $3.0(2 \cdot 5-3 \cdot 42)$ & $3 \cdot 17(2 \cdot 69-3 \cdot 66)$ & $3 \cdot 12(2 \cdot 52-3.54)$ & $2.94(2 \cdot 38-3.66)$ & $3.30(2 \cdot 77-4 \cdot 02)$ \\
\hline
\end{tabular}

Values are median (IQR) $(\mathrm{n}=20)$. Median (IQR) plasma $\mathrm{D}$-dimer values for population controls $(\mathrm{n}=158) 76 \mathrm{mg} / \mathrm{dl}(53-103)$. Median (IQR) plasma fibrinogen values for population controls $(\mathrm{n}=158) 2.6 \mathrm{~g} / 1(2.24-2.99) \mathrm{P}<0.05 ; \star \star \mathrm{P}<0.0001 v$ prewarfarin levels. (Two sample paired Wilcoxon test.)

plasma fibrinogen (median difference $0.08 \mathrm{~g} / \mathrm{l}$; $95 \% \mathrm{CI}-0.52$ to $0.77, \mathrm{P}=0.73$ ), $\mathrm{D}$-dimer (median difference $-34 \mathrm{ng} / \mathrm{ml} ; 95 \%$ CI -114 to $21 \cdot 0, P=0.25$ ), or $\mathrm{vWF}$ (median difference $2 \mathrm{IU} / \mathrm{dl} ; 95 \% \mathrm{CI}-35$ to 41 , $P=$ not significant levels between patients of groups 3 (aspirin alone) and 1 (table 2) (Mann-Whitney test).

There was a significant positive correlation between plasma fibrin D-dimer and vWF levels in patients of groups 1 and 3 (Spearman $P=0.52, P<0.001$ ), but no significant correlations between plasma fibrinogen and $\mathrm{D}$ dimer $(r=0 \cdot 18, \mathrm{P}=0 \cdot 18)$ or $\mathrm{VWF}(r=0 \cdot 17$, $\mathrm{P}=0 \cdot 22)$.

\section{EFFECTS OF INTRODUCING WARFARIN} TREATMENT ON PLASMA FIBRINOGEN AND FIBRIN D-DIMER LEVELS

There was a significant reduction in median plasma fibrin D-dimer levels at 2 months after the introduction of warfarin $(181 \mathrm{ng} / \mathrm{ml} v 80$ $\mathrm{ng} / \mathrm{ml}$, paired Wilcoxon test, $\mathrm{P}<0.001$ ) (table 3). A median reduction of plasma fibrin D-dimer by $41 \%$ (95\% CI $32 \%$ to $64 \%$ ) was therefore achieved after 2 months of anticoagulation. Sequential plasma D-dimer results were also available for 16 patients at days 3, 5, and 7 after the introduction of war-
Figure 2 Plasma D-dimer and chronic atrial fibrillation. Group 1 , patients with atrial fibrillation not receiving antithrombotic treatment group 2, patients with atrial fibrillation receiving warfarin; group 3, patients with atrial fibrillation receiving aspirin.

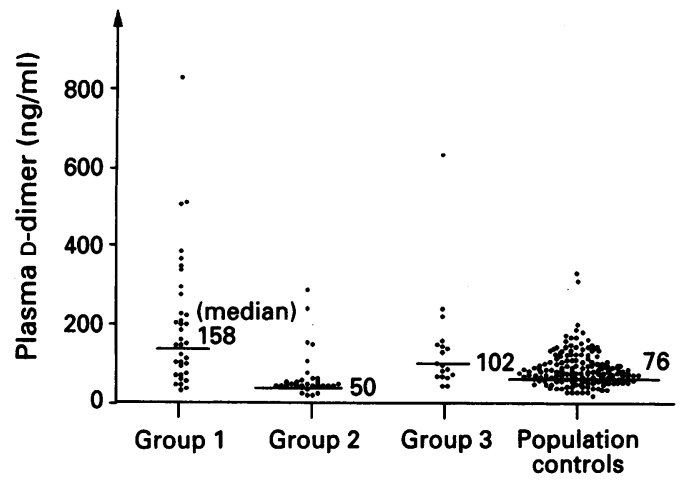

Figure 3 von Willebrand factor (vWF) and chronic atrial fibrillation. Group 1 patients with atrial fibrillation not receiving antithrombotic treatment group 2, patients with atrial fibrillation receiving warfarin; group 3, patients warfarin; group 3 , pan
with atrial fibrillation receiving aspirin. farin. There was a significant reduction in median plasma D-dimer level at day 3 after warfarin introduction $(181 \mathrm{ng} / \mathrm{ml} \quad v \quad 136$ $\mathrm{ng} / \mathrm{ml}$, paired Wilcoxon test, $\mathrm{P}=0.02$ ) compared with that at baseline but not at days 5 $(173 \mathrm{ng} / \mathrm{ml}, \mathrm{P}=0.75)$ or $7(130 \mathrm{ng} / \mathrm{ml}, \mathrm{P}=$ $0.07)$. There were no significant changes in plasma fibrinogen levels at any of the sampling points following the introduction of warfarin treatment (table 3 ). There was also no significant correlation between plasma fibrinogen or fibrin D-dimer levels and INR levels at any of the sampling points (data not shown).

INFLUENCE OF UNDERLYING PATHOLOGY, LEFT ATRIAL SIZE, AND LEFT VENTRICULAR FUNCTION

As there was no statistically significant difference in plasma fibrinogen, VWF, and fibrin Ddimer levels between patients with and without aspirin treatment (but not on warfarin) in the cross-sectional study (groups 1 and 3), further subgroup analysis was performed on the 56 patients in these two groups to study the influence of underlying pathology, atrial size, and left ventricular function.

There were no significant differences in plasma fibrinogen, D-dimer, or vWF levels when patients with valve disease or ischaemic heart disease were compared with those with no obvious aetiology (Mann-Whitney test, $P$ $=$ not significant). Technically adequate echocardiographic data were available for 52 patients not receiving warfarin (groups 1 and 3 ) and were related to plasma fibrinogen, vWF, and D-dimer. There were no significant correlations among left atrial dimensions, left atrial volume (ellipsoid), and left ventricular fractional shortening, and plasma fibrinogen, vWF, or fibrin D-dimer levels. There were also no differences in plasma fibrinogen (median difference $0.18 \mathrm{~g} / \mathrm{l} ; 95 \% \mathrm{CI}-0.38$ to $0.95, P=0.48$ ) or fibrin $\mathrm{D}$-dimer levels (median difference $-25 \mathrm{ng} / \mathrm{ml} ; 95 \% \mathrm{CI}-76$ to $47, P=0.29$ ) when patients with a left atrial dimension $>4.2 \mathrm{~cm}$ were compared in those with a left atrial dimension of $<4 \cdot 2 \mathrm{~cm}$. Plasma levels of vWF were higher, however in patients with a left atrium of $>4.2 \mathrm{~cm}$ than in those with a left atrium $<4.2 \mathrm{~cm}$ (median difference $42 \%$; $95 \%$ CI 3 to $87, P=0.045$ ). There were no differences in plasma fibrinogen (median difference $0.29 \mathrm{~g} / 1 ; 95 \% \mathrm{CI}$ -0.25 to $1.06, P=0.34$ ), fibrin $\mathrm{D}$-dimer levels (median difference $23 \mathrm{ng} / \mathrm{ml} ; 95 \% \mathrm{CI}-29$ to $112, \mathrm{P}=0.47$ ) or $\mathrm{vWF}$ (median difference $14 \% ; 95 \% \mathrm{CI}-32$ to $63, \mathrm{P}=0.49$ ) when patients with a left ventricular fractional shortening of $<25 \%$ were compared in those with normal fractional shortening ( $>25 \%)$. 
Stepwise multiple regression analysis with plasma fibrin $\mathrm{D}$-dimer as the dependent variable demonstrated that when age, left atrial volume (ellipsoid), right atrial volume, left ventricular fractional shortening, smoking habit, body mass index, and plasma fibrinogen were considered, only body mass index was a significant predictor for plasma fibrin $\mathrm{D}$ dimer $(P<0.05)$. Similar stepwise multiple regression analysis with plasma fibrinogen and vWF as dependent variables revealed no significant prediction by these variables.

\section{Discussion}

This study demonstrates that plasma levels of fibrinogen, fibrin D-dimer, and VWF are increased in patients with chronic atrial fibrillation not taking antithrombotic treatment compared with those of population sample controls in sinus rhythm. Increases of these three variables have also been reported in patients with atherosclerosis, ${ }^{5}$ in whom they are predictive of cardiovascular events ${ }^{61415}$; hence they may be markers of a prothrombotic state in patients with atrial fibrillation. Increased levels of plasma fibrinogen may promote thromboembolic events by increasing the tendency to thrombosis (as suggested by the weak positive correlation with plasma $\mathrm{D}$-dimer levels in the whole group), or by increasing flow disturbance through its rheological effects in increasing red cell aggregation and blood viscosity in areas of stasis, for example, in the dilated atria in patients with atrial fibrillation. Increased $\mathrm{vWF}$ and D-dimer levels suggest increased endothelial disturbance and increased fibrin turnover respectively; these results confirm two previous smaller studies $^{89}$ and suggest two further mechanisms for thrombogenesis.

Smoking is associated with increases in plasma fibrinogen, vWF, and D-dimer, ${ }^{35}$ however, our results were not attributable to an excess of smokers in the atrial fibrillation group (table 1). The increases in plasma fibrinogen, vWF, and fibrin D-dimer were not related to underlying cardiac disease, with no statistically significant differences between patients with an underlying cause for atrial fibrillation (ischaemic heart disease, valve disorders) and those with no obvious cause, although a trend towards higher median plasma fibrinogen levels in patients with ischaemic heart disease was noted. Observations in the large local population survey (the second north Glasgow monitoring trends and determinants in cardiovascular disease study) have shown that those with ischaemic heart disease and sinus rhythm had relatively minor increases in plasma fibrinogen, vWF, and D-dimer levels compared with those in patients with no ischaemic heart disease and sinus rhythm ${ }^{10}$ (Lowe et al and unpublished observations). Patients in group 3 (aspirin only) had a higher mean age than patients in other groups, however, we feel that the slightly older, but small numbers of patients in group 3 had no significant effect on the results, as age was not a significant pre- dictor for these factors in our patients using multiple regression analysis. In addition, there was only a relatively small difference between levels of these markers between the adjacent age bands in our population survey.

The increases in plasma fibrinogen, vWF, and fibrin D-dimer are also unlikely to be related to a diurnal variation in levels or an effect of physical activity (especially on fibrinogen levels), as this has been explored in our local population survey, where no effect of a diurnal variation was seen and the effect of physical activity was small ${ }^{10}$ (Lowe et al, and unpublished publication). We therefore feel that physical activity alone cannot explain high levels of markers in our patients. Another limitation of this study is that the precise duration of known atrial fibrillation in patients from the cross-sectional study cannot be determined. We were satisfied, however, that patients studied had chronic atrial fibrillation as the patients had atrial fibrillation documented on at least two visits to their general practitioner, hospital, or our clinic. Our objective was to study patients when referred to us or when detected at follow-up in our clinic and in addition, it was considered unethical to withold antithrombotic treatment once such patients were identified. We carefully avoided studying patients with any concomitant illnesses (including bleeding, infection, and any acute cardiovascular syndromes) that may have influenced levels of these markers.

Warfarin is also not known to influence any underlying pathological process and therefore fibrinogen or vWF levels would not have been expected to be different between the groups with and without warfarin treatment, as demonstrated in both the cross-sectional and longitudinal arms of our study. We therefore suggest that it is atrial fibrillation itself which is the major contributory factor to the increased thrombotic tendency as reflected by the raised fibrinogen, vWF, and D-dimer levels. There was no significant correlation of these variables with atrial size or left ventricular function.

An important question therefore is why is atrial fibrillation associated with an increased plasma fibrinogen or vWF level. It is unlikely that the increased fibrinogen or vWF levels are a secondary response to increased clot or thrombus formation, as in such cases D-dimer levels would have been raised in the subgroups with and without warfarin. Abnormalities in cardiac blood flow (for example sluggish, slow flow within the atria) may be partly responsible, resulting in flow abnormalities and adding to endothelial disturbance (causing raised vWF) in the pulmonary vasculature. This latter effect may stimulate lung macrophages to produce hepatocyte stimulating factor (now known to be interleukin 6), increasing liver synthesis of fibrinogen (in a similar manner to that of smoking). ${ }^{16}$ Further studies investigating the mechanisms by which fibrinogen and vWF synthesis are increased are needed. The precise mechanism for the increased plasma fibrinogen and vWF levels is therefore uncertain. 
Our study shows that vWF levels were significantly increased in patients with chronic atrial fibrillation irrespective of anticoagulant treatment. Our findings therefore suggest a role for VWF in the pathogenesis of thromboembolic risk in patients with chronic atrial fibrillation. As VWF is a marker of endothelial (which may extend to endocardial) disturbance, this is consistent with Virchow's observation of the importance of the vessel wall in thrombogenesis (Virchow's triad). ${ }^{17}$ The findings of a modest correlation between plasma vWF and fibrin D-dimer levels in patients not receiving warfarin $(r=0.52, \mathrm{P}<0.001)$, and higher vWF levels in patients with a large left atrium $(>4.2 \mathrm{~cm})$ are in support of this hypothesis.

As plasma D-dimer is a marker of crosslinked fibrin turnover, our finding that plasma levels of $\mathrm{D}$-dimer were significantly increased in patients with atrial fibrillation (without any antithrombotic treatment) compared with that in controls in sinus rhythm suggests that there is ongoing intravascular fibrin formation in these patients, which is consistent with two previous small studies. ${ }^{89}$ In a small study of patients with atrial fibrillation and mitral stenosis increased levels of other markers of a thrombotic state (fibrinopeptide A, thrombinantithrombin III complex) have been demonstrated within the left atria of such patients. ${ }^{18}$ As increased plasma fibrin D-dimer levels suggest ongoing intravascular fibrin turnover (and possibly early thrombus formation), such patients with chronic atrial fibrillation not receiving antithrombotic treatment are at increased risk of thrombogenesis. If warfarin or aspirin effectively prevented intravascular fibrin and thrombus formation, then D-dimer levels would be expected to be lower in patients receiving warfarin or aspirin treatment. This is indeed clearly demonstrated for warfarin in our study which found that the median plasma D-dimer level was about two thirds lower on warfarin treatment, which is consistent with the finding that warfarin reduces thromboembolic risk by about two thirds based on randomised clinical trials. ${ }^{10}$

As anticoagulation treatment is not without risk, clinicians contemplating antithrombotic treatment for prophylaxis against thromboembolic events in patients with atrial fibrillation have to balance the benefit of risk reduction against the risks of haemorrhage with warfarin. ${ }^{1019}$ To be able to select patients who would most benefit from warfarin would be a major advance. Our study suggests that the measurement of plasma fibrin D-dimer may identify those with the highest levels of intravascular turnover, who theoretically may be at highest thromboembolic risk. Large scale prospective studies are required to test this hypothesis and may be to assess the predictive value of plasma fibrinogen and vWF levels for thromboembolic complications. The apparent lack of effect of warfarin (or aspirin) on plasma fibrinogen or VWF levels suggests that they could be used as risk markers even in patients taking antithrombotic medication.
In our longitudinal study we also observed a sequential reduction of plasma fibrin $\mathrm{D}$ dimer after the introduction of warfarin, which was significant at 2 months. The lack of a significant decrease in median plasma Ddimer at days 5 and 7 may in part reflect the fact that the patients were not yet stabilised on warfarin. This result confirms the effectiveness of warfarin in reducing intravascular fibrin turnover. The lack of correlation between plasma fibrin D-dimer and the INR value suggests that $\mathrm{D}$-dimer levels might be useful supplements to INR estimation, measuring antithrombotic effect in vivo rather than reduction in vitamin $\mathrm{K}$ dependent clotting factors. The quantification of the INR is thus not necessarily directly related to the efficacy of warfarin in reducing thrombogenesis; the INR is a measure of the effect of warfarin on clotting factors (Factors II, VII, IX and X), while the reduction in fibrin $\mathrm{D}$-dimer may reflect fibrin turnover and the efficacy of reducing the production/formation of fibrin or thrombus.

Patients with atrial fibrillation receiving aspirin had a lesser (about one third) reduction in plasma $\mathrm{D}$-dimer levels compared with that of patients not receiving antithrombotic treatment. This difference was not statistically significant. This may be a reflection of either a lower antithrombotic efficacy of aspirin, or the smaller number of patients within this group. In addition, the mechanisms of action of aspirin and warfarin differ. Larger studies of aspirin are therefore required to assess its effect on D-dimer levels and also its effects on risk reduction of thromboembolism in patients with atrial fibrillation: the current estimate is a risk reduction of about $25 \%)^{10}$

In conclusion, our results show significantly increased median plasma fibrinogen and vWF levels in patients with chronic atrial fibrillation, which seem to be unaffected by aspirin or warfarin. Plasma D-dimer levels were also raised in patients with chronic atrial fibrillation not receiving antithrombotic treatment, suggesting increased intravascular thrombogenesis in such patients. In patients receiving warfarin treatment there was a reduction of circulating fibrin D-dimer levels, suggesting that warfarin was effective in preventing intravascular fibrin turnover and thrombogenesis; this is the first demonstration that the introduction of warfarin significantly reduces a laboratory index of thrombogenesis. These results also suggest three possible thrombotic markers to assess those patients with atrial fibrillation who may be at high risk of thrombogenesis; plasma fibrin D-dimer also merits assessment as a possible marker of ongoing thrombogenesis, and as a measure of reduction in thrombotic risk in patients given warfarin. This may open the possibility of identifying patients with atrial fibrillation who are at high risk of thromboembolism, and might aid decision making when warfarin treatment is being considered. Further large prospective studies are required to explore these possibilities. 
We thank $\mathrm{Dr}$ AJ Lee and Professor $H$ Tunstall-Pedoe, Cardiovascular Epidemiology Unit, University of Dundee, for permission to use data from the second Glasgow MONICA survey, Dr AP Rae and Dr KJ Hogg for allowing us to study their patients, Dr MJ Metcalfe for helpful comments during the preparation of this manuscript, and Dr J Curral for the preparation of this
valuable statistical advice.

1 Meade TW, North WRS, Chakrabarti R, Stirling Y, Haines AP, Thompson SG. Haemostatic function and cardiovascular death: early results of a prospective study. Lancet 1980;i:1050-4.

2 Wihelmsen L, Svardsudd K, Korsan-Bengtsen K, Larsson $\mathrm{B}$, Welin L, Tibblin G. Fibrinogen as a risk factor for stroke and myocardial infarction. $N$ Engl $₹$ Med 1984; 311:501-5.

3 Ernst E, Resch KL. Fibrinogen as a cardiovascular risk factor: a meta-analysis and review of the literature. Ann Intern Med 1993;118:956-63.

4 Uchiyama S, Takeuchi M, Osawa M, Kobayashi I, Maruyama S, Aosaki M, et al. Platelet function tests in Maruyama S, Aosaki $M$, et al. Platelet function tests in thrombo

5 Smith FB, Lowe GDO, Fowkes FG, Rumley A, Rumley AG, Donnan PT, et al. Smoking, haemostatic factors and lipid peroxides in a population case control study of peripheral arterial disease. Atherosclerosis 1993;102: 155-62.

6 Fowkes FGR, Lowe GDO, Housley E, Rattray A, Rumley A, Elton RA, et al. Cross-linked fibrin degradation products, progression of peripheral arterial disease, and risk of coronary heart disease. Lancet 1993;342:84-6.

7 Hunt FA, Rylatt DB, Hart RA, Bundesen PG. Serum crosslinked fibrin (XDP) and fibrinogen/fibrin deoradation products (FDP) in disorders associated with activa tion of the coagulation or fibrinolytic systems. $\mathrm{Br} F$ Haematol 1985;60:715-22.
8 Gustafsson C, Blomback M, Britton M, Hamsten A, Svensson J. Coagulation factors and the increased risk of stroke in nonvalvular atrial fibrillation. Stroke 1990; 21:47-51.

9 Kumagai K, Fukunami M, Ohmori M, Kitabatake A Kamada T, Hoki N. Increased intracardiovascular clotting in patients with chronic atrial fibrillation. $₹ \mathrm{Am}$ Coll ting in patients with chro

10 Lowe GDO. Antithrombotic treatment and atrial fibrillation. $B M F$ 1992;305:1445-6.

11 Lowe GDO, Lee AJ, Rumley A, Smith WCS, TunstallPedoe H. Epidemiology of haematocrit, white cell count, red cell aggregation and fibrinogen: the Glasgow MONICA study. Clin Hemorheol 1992;12:535-44.

12 Sanfilippo AJ, Abascal VM, Sheehan M, Oertel LB, Harrigan P, Hughes RA, et al. Atrial enlargement as a consequence of atrial fibrillation. A prospective echocardiographic study. Circulation 1990;82:792-7.

13 Sahn DJ, DeMaria A, Kisslo J, Weyman A. Recommendations regarding quantitation in $M$-mode echocardiography: Results of a survey of echocardiographic measurements. Circulation 1978;58:1072-83.

14 Jansson $J \mathrm{H}$, Nilsson TK, Johnson $O$. von Willebrand factor in plasma: a novel risk factor for recurrent factor in plasma: a novel risk factor for recurrent 351-5.

15 Banerjee AK, Pearson J, Gilliland EL, Goss D, Lewis JD, Stirling Y, et al. A six year prospective study of fibrinogen and other risk factors associated with mortality in stable claudicants. Thromb Haemost 1992;68:261-3.

16 Ritchie DG, Levy BA, Adams MA, Fuller GM. Regulation of fibrinogen synthesis by plasmin-derived fragments of fibrinogen and fibrin: an indirect feedback pathway. Proc Natl Acad Sci USA 1982;79:1530-4.

17 Virchow R. Gesammelte abhandlungen zur wissenschaftichen medtzin. Frankfurt: Medinger Sohn, 1856:219-732.

18 Yamamoto $K$, Ikeda U, Shimada $K$. A hypercoagulable state in the left atrium of patients with mitral stenosis. N Engl ₹ Med 1993;328:1043-4.

19 Ramsay LE. Warfarin in chronic atrial fibrillation. Lancet 1993;341:1376-7. 